\title{
Statistical Validation of Heart Rate Measurement Using Modulated Magnetic Signature of Blood with Respect to Electrocardiogram
}

\author{
Chee Teck Phua, Gaelle Lissorgues, Boon Chong Gooi, and Bruno Mercier
}

\begin{abstract}
Heart rate (HR) is an important human physiological marker commonly used for the understanding of an individual's physical health. This paper adopts and outlines the magnetic method of non-invasive acquisition of blood pulse using the disturbance created by blood flowing through a localized magnetic field (i.e. Modulated Magnetic Signature of Blood - MMSB). The measurement setup is designed to acquire the MMSB and a gold standard instrumentation, Electrocardiogram (ECG) for 20 healthy subjects (10 males and 10 females, aged 18-22). Two independent measurements were conducted for each subject resulting in $40 \mathrm{HR}$ measurements collected. Four statistical tests were applied to validate the instantaneous HR measured from MMSB waveform with respect to the ECG waveform. First, the Pearson Correlation and Coefficient of Determination was applied on the measured HR and both MMSB and ECG methods of measurements were positively correlated $(R=0.99)$ with perfectly linear relationship ( $<1 \%$ scores in the data due to error). Secondly, the Paired Student's t-test and the Wilcoxon Signed-Rank Test were applied on the measured HR and they showed no significant difference (at $95 \%$ limits of agreement) for the mean and median calculated from HR measured from the two methods. Lastly, the Bland and Altman Test is applied on the measured HR and the $\pm 2 *$ Standard Deviation (SD) is derived as 0.48 blood pulse per minute (bpm). This is not significant for measurement of resting HR for healthy individual, who has a typical resting $H R$ of $\pm 1 \mathrm{bpm}$. The result obtained from the Bland and Altman Test affirms accuracy of MMSB to be used as an alternative to ECG system for HR measurements. Results obtained from all the four statistical tests are coherent and supports the use of MMSB as a viable alternative to ECG for measuring resting HR for healthy individuals.
\end{abstract}

Index Terms -Electrocardiogram, magnetic blood pulse, modulated magnetic signature of blood, statistical validation for heart rate measurement

\section{INTRODUCTION}

With the advancement of bioelectronics, portable health monitoring devices are able to provide continuous monitoring of physiological signs to assess the health of an individual. One such physiological sign is the heart rate (HR), which is commonly used by hospitals and elderly care centers to monitor the health conditions of their patients.

Manuscript received March 1, 2012; revised March 29, 2012. This work was supported by the Nanyang Polytechnic of Singapore.

Chee Teck Phua is with the Nanyang Polytechnic of Singapore, 180 Ang Mo Kio Avenue 8, Singapore 569830 (phone: +65-6550-0703; fax: +65 6452 0400; e-mail: phua_chee_teck@nyp.gov.sg).

Gaelle Lissorgues and Bruno Mercier are with ESIEE - ESYCOM University Paris Est, France (e-mail: lissorgg@esiee.fr, b.mercier@esiee.fr).

Boon Chong Gooi is with the Nanyang Polytechnic of Singapore (e-mail: gooi_boon_chong@nyp.gov.sg).
Current methods of heart or pulse rate acquisition can be classified into electrical [1], optical [2], microwave [3], acoustic [4], mechanical [5] or magnetic [6-7] means. Amongst these methods, the Electrocardiogram (ECG), which records the electrical conductivity of the heart, serves as the gold standard for the non-invasive HR measurement [8] and diagnosis of arrhythmias and conduction disturbances.

This paper will review the recent work on magnetic blood pulse sensing [7] and adopt this measurement approach to measure the magnetic disturbance created by pulsatile flow of blood (i.e. Modulated magnetic signature of blood MMSB) through an applied constant magnetic flux. The use of magnetic flux in MMSB enables the acquisition of blood pulse for HR measurement without the need for a good electrical or optical contact.

In medical measurement, statistical methods are applied to compare a new method for HR measurement (i.e. the MMSB method) with an established one (i.e. ECG). The objective of these comparisons is to determine whether these two methods can be used interchangeably or the new method can replace the established one [9]-[14].

In this paper, the instantaneous HR measured using MMSB will be statistically validated with respect to ECG, a gold standard instrumentation. In this validation, four statistical tests will be applied on the instantaneous HR measured. These statistical tests are Pearson Correlation and Coefficient of Determination [15], Paired Student's t-test [16], Wilcoxon Signed-rank Test [17] and Bland and Altman Test [18]-[19].

The results obtained from these each of these statistical tests aims to validate the use of MMSB as a viable alternative for measuring $\mathrm{HR}$ in resting condition for healthy individuals. More importantly, the combine effects of the four statistical tests will provide the affirmation that MMSB can be used in place of ECG for resting HR measurements for healthy adults.

\section{Modulated Magnetic Signature OF BloOd}

The concept of magnetic blood pulse measurement [7] applies a uniform magnetic field in the vicinity of the major artery as shown in Fig. 1. As the heart pumps, blood flows through the applied magnetic field in a pulsatile nature that will disturb the magnetic field. The creation of the unique magnetic disturbance is dependent on the volumetric changes in blood and can be acquired using a Giant Magnetoresistance (GMR) based magnetic sensor operating at room temperature as shown in Fig. 1.

Magnetic flux is able to propagate through a layer of 
material such as fabric, body-fluids and environmental contaminants. As such, the use of magnetic flux in MMSB supports the acquisition of blood pulse without the need for an electrical or optical contact with the skin.

The placement of the sensor and magnet on the wrist and heel are illustrated as shown in Fig. 2 [7]. A typical MMSB waveform captured by the sensor on these placements is shown in Fig. 3.

Using Fig. 2 (a), a button magnet of magnetic field strength 0.1 Tesla is placed on the wrist with a GMR sensor from NVE (e.g. AAH-004). Such a placement will produce a typical MMSB waveform (refer to Fig. 3) that shows a well defined signal that is highly correlated to the activities of the heart [7].

Through the extraction of the time difference between two peaks, instantaneous HR can be easily measured from the MMSB waveform based on the time difference between the two consecutive peaks. This is the approach for HR measurements was reported in [7] and will be used in this paper.

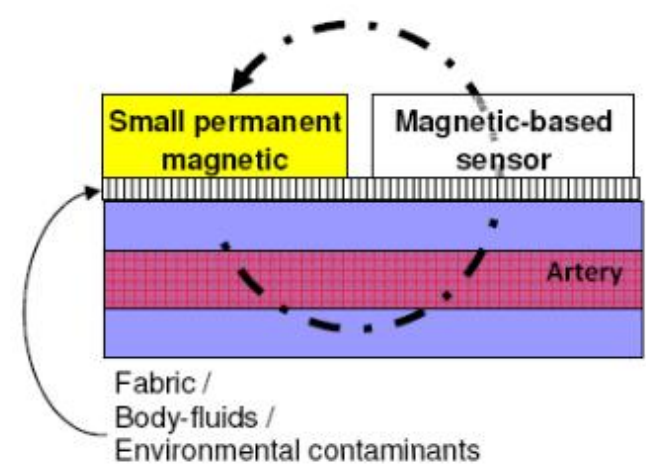

Fig. 1. Cross-sectional view of the experimental setup to acquire MMSB [7]

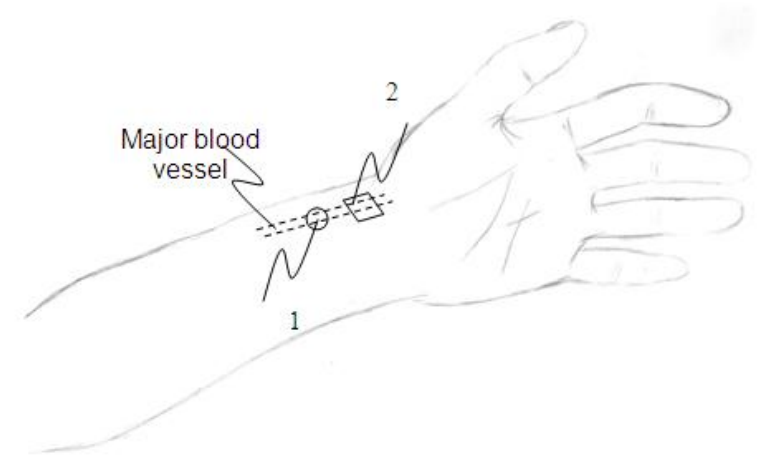

(a) Placement of sensor and magnet on the wrist

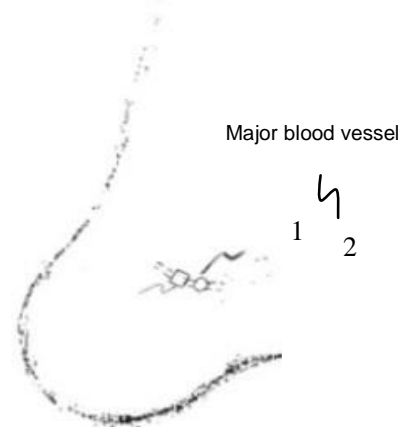

(b) Placement of sensor and magnet on the heel

Fig. 2. Examples of MMSB acquisition based on the anatomy of human and the relative positioning of the magnet (1), sensor(2) and a major blood vessel [7]

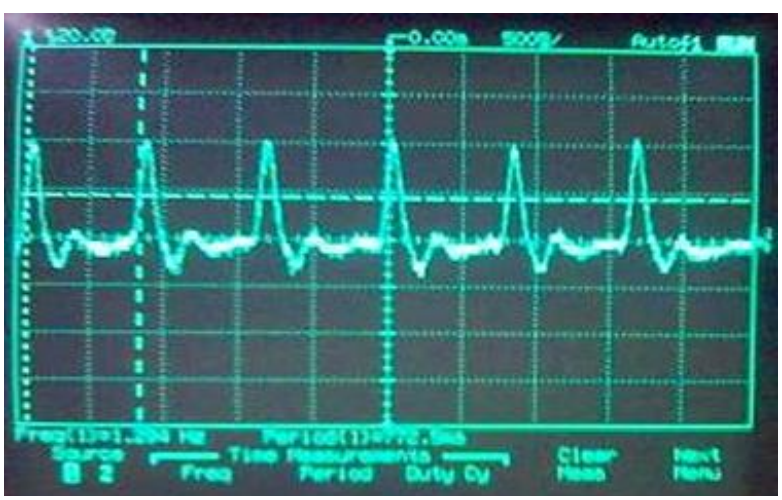

Fig. 3. Waveform captured from the sensor output using oscilloscope [7]

\section{SETUP For HeART RATE MEASUREMENTS}

The setup for concurrent heart wave acquisition using ECG and MMSB is shown in Fig. 4.

For the ECG measurements, three electrodes were placed on each subject. These electrodes form the Eithoven triangle [20] as shown in Fig. 4. The electrode placed below the heart serves as the reference for the zero potential of the electrodes placed on the right and left shoulder. Using the difference between the electrodes placed on the right and left shoulder, the bipolar ECG waveform (i.e. Lead I equivalent) is obtained as shown in Fig. 5 (a).

MMSB data is concurrently acquired from the wrist of the subject using the placement of sensor and magnet as shown in shown in Fig. 2 (a). The MMSB waveform obtained from the wrist is shown in Fig. 5 (b).

ECG and MMSB waveform were concurrently obtained using g.USBamp, (from g.tec Medical Engineering GMBH, Austria) at a sampling rate of $1 \mathrm{KHz}$ and amplitude resolution of 24 bits over $+250 \mathrm{mV}$. The use of $1 \mathrm{KHz}$ sampling rate is used to provide good time resolution for statistical validation of HR measurement.

Using the measurement setup in Fig. 4, measurements were conducted on 20 healthy subjects (i.e. 10 males and 10 females, aged 18-22) with no known medical history or cardiovascular disorder.

Before the start of each measurement, each subject was given a 5-minute rest interval. During this period, the subject will be seated with minimum physical activity.

After which, ECG electrodes and MMSB sensor and magnet were placed on the subject while he was in a resting state. Measurement was commenced after the waveforms for both ECG and MMSB are stabilized (typically 1-2 minutes upon the placement of ECG electrodes and MMSB sensor).

For each measurement, 2 minutes of MMSB and ECG waveform was recorded for HR extraction. The ECG and MMSB waveform are automatically saved into the computer harddisk through the MATLAB interface.

Upon the completion of each measurement, the MMSB and ECG waveforms for the subject were plotted using the MATLAB build-in graph function.

The instantaneous HR for ECG measurement was obtained from the time difference measured between two consecutive $\mathrm{R}$ peaks of ECG waveform (refer to Fig. 5(a)). Using the marker and peak search functions from MATLAB, HR for each subject was manually extracted by subtracting the time difference between two consecutive $\mathrm{R}$ peaks. 
Similarly, instantaneous HR was measured from the MMSB waveform by locating the corresponding peaks (with respect to the $\mathrm{R}$ peaks in the ECG waveform) of two consecutive MMSB waveforms as shown in Fig. 5 (b).

Two measurements were conducted on each subject on separate days. Therefore, each measurement was taken to be independent and the waveform collected will result in a total of 40 resting ECG and MMSB HR measurements.

The instantaneous HR in blood pulse per minute (bpm), calculated from the ECG and MMSB waveform (refer to Fig. 5 ), were tabulated as shown in Table I. In order to compute the differences between instantaneous HR measured from the ECG and MMSB waveform, the time resolution based on the sampling rate of $1 \mathrm{KHz}$ (i.e. 3 decimal places) is recorded to provide the accuracy needed for statistical validation.

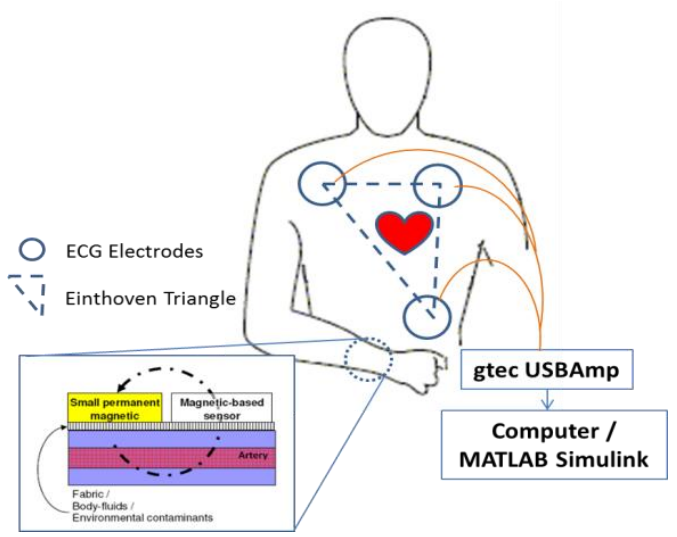

Fig. 4. HR acquisition using ECG and MMSB concurrently

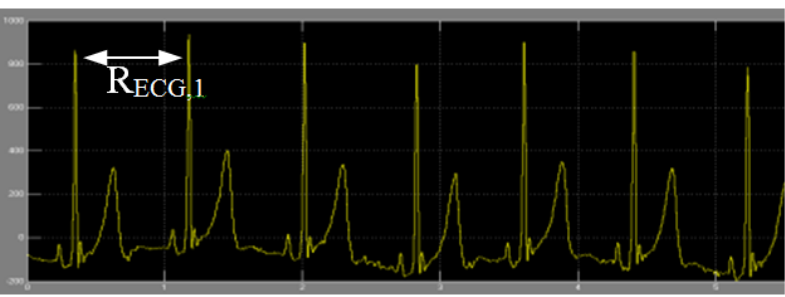

(a) Instantaneous HR from ECG signal

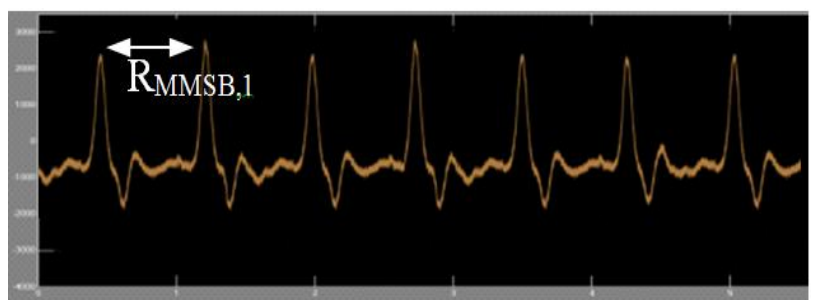

(b) Instantaneous HR from MMSB signal

Fig. 5. Extraction of instantaneous HR from ECG and MMSB waveforms

TABLE I: HEART RATE DATA COLLECTED FOR 40 MEASUREMENTS

\begin{tabular}{|c|c|c|c|}
\hline Measurement & $\begin{array}{c}\text { Gold Standard } \\
\text { (ECG) bpm }\end{array}$ & $\begin{array}{c}\text { MMSB } \\
\text { Sensor bpm }\end{array}$ & $\begin{array}{c}\text { \% of } \\
\text { difference } \\
\text { w.r.t. ECG }\end{array}$ \\
\hline 1 & 78.174 & 78.271 & -0.12 \\
\hline 2 & 77.857 & 77.994 & -0.18 \\
\hline 3 & 78.174 & 78.694 & -0.67 \\
\hline 4 & 75.084 & 74.912 & 0.23 \\
\hline 5 & 78.976 & 79.033 & -0.07 \\
\hline 6 & 78.094 & 78.311 & -0.28 \\
\hline 7 & 89.238 & 89.706 & -0.52 \\
\hline
\end{tabular}

\begin{tabular}{|c|c|c|c|}
\hline Measurement & $\begin{array}{l}\text { Gold Standard } \\
\text { (ECG) bpm }\end{array}$ & $\begin{array}{c}\text { MMSB } \\
\text { Sensor bpm }\end{array}$ & $\begin{array}{c}\% \text { of } \\
\text { difference } \\
\text { w.r.t. ECG }\end{array}$ \\
\hline 8 & 95.557 & 95.654 & -0.10 \\
\hline 9 & 70.475 & 70.734 & -0.37 \\
\hline 10 & 76.540 & 76.747 & -0.27 \\
\hline 11 & 58.945 & 58.712 & 0.40 \\
\hline 12 & 56.148 & 56.659 & -0.91 \\
\hline 13 & 83.160 & 82.810 & 0.42 \\
\hline 14 & 93.071 & 92.973 & 0.11 \\
\hline 15 & 77.426 & 77.251 & 0.23 \\
\hline 16 & 73.121 & 73.253 & -0.18 \\
\hline 17 & 80.003 & 79.906 & 0.12 \\
\hline 18 & 80.802 & 80.619 & 0.23 \\
\hline 19 & 80.548 & 80.548 & 0.00 \\
\hline 20 & 89.759 & 89.445 & 0.35 \\
\hline 21 & 80.170 & 80.674 & -0.63 \\
\hline 22 & 88.417 & 88.113 & 0.34 \\
\hline 23 & 87.264 & 87.363 & -0.11 \\
\hline 24 & 92.918 & 92.838 & 0.09 \\
\hline 25 & 84.998 & 84.783 & 0.25 \\
\hline 26 & 100.553 & 100.160 & 0.39 \\
\hline 27 & 75.047 & 74.966 & 0.11 \\
\hline 28 & 80.911 & 80.911 & 0.00 \\
\hline 29 & 80.488 & 80.488 & 0.00 \\
\hline 30 & 71.390 & 71.390 & 0.00 \\
\hline 31 & 91.888 & 92.215 & -0.36 \\
\hline 32 & 97.680 & 97.583 & 0.10 \\
\hline 33 & 57.107 & 57.128 & -0.04 \\
\hline 34 & 57.982 & 58.055 & -0.13 \\
\hline 35 & 99.738 & 100.063 & -0.33 \\
\hline 36 & 93.809 & 94.038 & -0.24 \\
\hline 37 & 94.268 & 94.096 & 0.18 \\
\hline 38 & 88.367 & 88.774 & -0.46 \\
\hline 39 & 65.746 & 65.793 & -0.07 \\
\hline 40 & 69.851 & 69.930 & -0.11 \\
\hline
\end{tabular}

\section{STATISTICAL VALIDATION FOR ASSESSING AGREEMENT BetweEn Two Methods of ClinicAl MEASUREMENT}

\section{A. Pearson Correlation and Coefficient of Determination}

Pearson correlation coefficient (direction of relationship) and the Coefficient of Determination tests (strength of relationship) is used in the validation of the measurement outputs (i.e. instantaneous HR) of an alternative instrument (i.e. MMSB) against a "gold standard" (i.e. ECG) to ascertain the nature of relationship between two sets of scores [21]. The expression for Pearson's Correlation Coefficient is 
shown in (1).

$$
r_{X Y}=(\Sigma X Y-(((\Sigma X)(\Sigma Y)) / n)) /\left(\sqrt{\left(S S_{X}\right)\left(S S_{Y}\right)}\right)
$$

where

$\mathrm{r}_{\mathrm{XY}}=$ Pearson correlation coefficient

$\mathrm{X}=$ instantaneous HR for MMSB

$\mathrm{Y}=$ instantaneous $\mathrm{HR}$ for $\mathrm{ECG}$

$\mathrm{n}=$ number of pairs of data (i.e. 40$)$

$S S_{X}=\Sigma X^{2}-\left((\Sigma X)^{2} / n\right)$

$S S_{Y}=\Sigma Y^{2}-\left((\Sigma \mathrm{Y})^{2} / n\right)$

Using the formula for Pearson's Correlation Coefficient (1), the Pearson correlation coefficient $\left(r_{X Y}\right)$ and the Coefficient of Determination $\left(\mathrm{r}_{\mathrm{XY}}{ }^{2}\right)$ were calculated as shown in Table II.

From the results shown in Table II, it can be observed that the HR data between MMSB and ECG was positively correlated (i.e. $r_{X Y}=+0.9998$ ). The corresponding coefficient of determination $\left(\mathrm{r}_{\mathrm{XY}}{ }^{2}\right)$ is 0.9996 .

These results (i.e. $r_{X Y}=0.9998$ and $r_{X Y}{ }^{2}=0.9998$ ) indicate that the relationship between the HR measured by both the MMSB and ECG methods was almost perfectly linear*. More importantly, the results show that $<1 \%$ of scores in the HR data from MMSB and ECG is due to error between the two measurements.

Using Table I, the instantaneous HR for MMSB and ECG measurements were plotted as shown in Fig. 6.

To understand the direction of relationship, a linear regression approach was adopted to approximate the linear equation to describe the two measurements. The linear equation obtained is shown in equation (2) with regression value $R^{2}=0.9996$ obtained between equation (2) and the data The $\mathrm{R}^{2}$ value being almost 1 indicates an almost perfect positive relationship between the measured data and the linear equation (2).

$$
\mathrm{Y}=0.9971 \mathrm{X}+0.2845
$$

where

$\mathrm{X}=$ instantaneous $\mathrm{HR}$ for MMSB

$\mathrm{Y}=$ instantaneous $\mathrm{HR}$ for $\mathrm{ECG}$

Based on the results obtained from this correlation study, it was concluded that the reproducibility of the HR measurement between the 2 devices is almost 1:1. The minute differences observable in this test was made possible through the resolution (i.e. up to 3 decimal places) provided by the sampling frequency of $1 \mathrm{KHz}$.

The reproducibility of measurement results is an important step forward as it forms the basis for any scientific validation to be meaningful.

TABLE II: CORRELATION TEST BETWEEN GOLD REFERENCE AND MMSB SENSOR

\begin{tabular}{|l|c|}
\hline \multicolumn{2}{|c|}{ Regression Statistics } \\
\hline Multiple $\mathrm{r}_{\mathrm{XY}}$ & 0.9998 \\
\hline $\mathrm{r}_{\mathrm{XY}}$ Square & 0.9996 \\
\hline Standard Deviation (SD) & 0.2440 \\
\hline 2xSD & 0.4880 \\
\hline
\end{tabular}

*If $\mathrm{r}=1.00$ there is a perfect positive relationship between the HR obtained from MMSB and ECG waveform (i.e. perfect match).

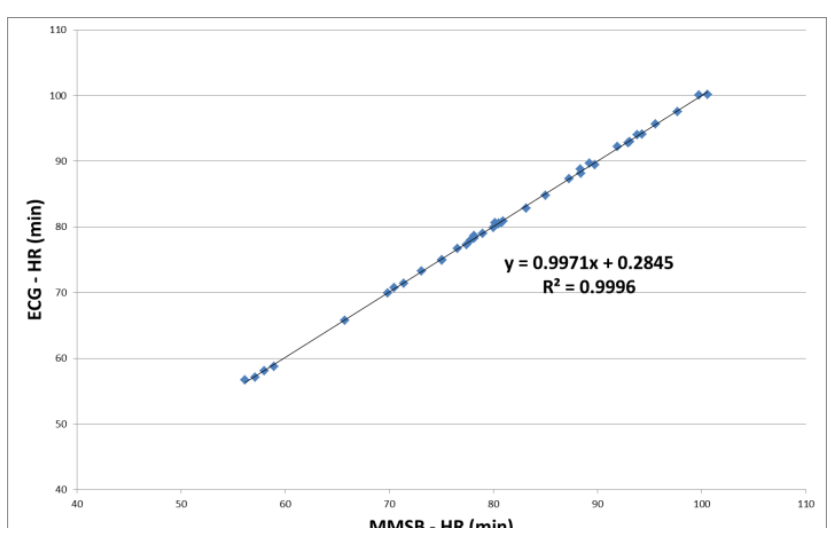

Fig. 6. Plot of HR measured from MMSB and ECG

\section{B. Paired Student's t-test}

The Paired Student's t-test is used as part of the scientific validation on the measurement outputs (refer to Table I) to establish the statistical significance of the differences in the means between the two sets of scores [22].

From the HR obtained in Table I, the paired t-test determines whether HR measured from MMSB and ECG differ from each other in a significant way under the assumptions that the paired differences are independent and follows a normal distribution (i.e. $\mathrm{P}^{\dagger}<0.05$ ).

The equation for obtaining the probability of obtaining the test statistic for the Paired Student t-test is shown in (3).

$$
P=(\bar{X}-\bar{Y}) / \sqrt{\left(s_{1}{ }^{2} / n_{1}+s_{2}{ }^{2} / n_{2}\right)}
$$

where

$\mathrm{P}=$ probability of obtaining a test statistic

$X=$ instantaneous HR obtained from MMSB

$Y=$ instantaneous HR obtained from ECG

$\bar{X}=$ mean of instantaneous HR obtained from MMSB

$\bar{Y}=$ mean of instantaneous HR obtained from ECG

$\mathrm{n}_{1}=\mathrm{n}_{2}=$ number of paired HR obtained from MMSB and ECG

$s_{1}{ }^{2}=$ variance of instantaneous HR obtained from MMSB = $\Sigma\left(X_{i}-\bar{X}\right)^{2} / n_{1}$

$s_{2}{ }^{2}=$ variance of instantaneous HR obtained from MMSB = $\Sigma\left(Y_{i}-\bar{Y}\right)^{2} / n_{2}$

The mean (i.e. $\bar{X}$ and $\bar{Y}$ ) and standard deviation (i.e. $\mathrm{s}_{1}$ and $s_{2}$ ) were calculated and plotted as shown in Fig. 4. Using (3), the equation for Paired Student t-test, $\mathrm{P}$ was calculated as 0.24 . This result shows that there was no significant difference between the means of the HR measured by both devices (Fig. 7).

In addition, this result of the statistical test suggested the difference between the two sets of measurement is statistically not significant.

Therefore, the results obtained from the Paired Student t-test supports the use of MMSB as a viable alternative to ECG for HR measurement.

\footnotetext{
${ }^{\dagger} \mathrm{P}$-value is the probability of obtaining a test statistic at least as extreme as the one that was actually observed, assuming that the null hypothesis is true. In this case a P-value less than 0.05 would result in the rejection of the null hypothesis at the $5 \%$ (significance) level. It means that if the HR is sampled on numerous occasions and interval estimates are made on each occasion, the resulting intervals would bracket the true HR parameter in approximately $95 \%$ of the cases. This is sufficient for typical clinical validation.
} 


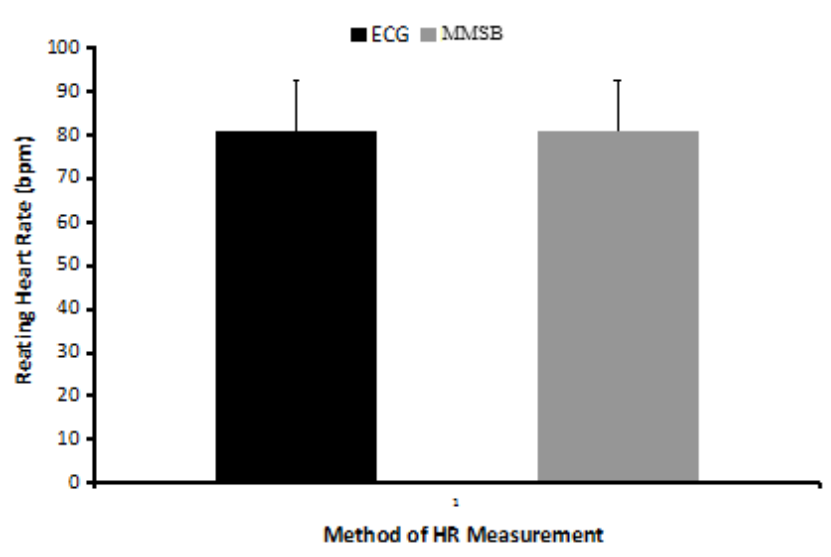

Fig. 7. Mean and standard deviation of HR measured with the ECG and MMSB sensors $(\mathrm{P}=0.24)$

\section{Wilcoxon Matched-Pairs Signed-Ranks Test}

The Wilcoxon Signed-Rank Test is used as part of the scientific validation to determine whether there is a statistical significance on the difference in the median between paired samples [23]-[24].

The Wilcoxon Signed-Rank test was applied on the HR measurement from Table I. The Null Hypothesis for the Wilcoxon Signed-Rank Test (W) states that there was no difference between the paired samples at $95 \%$ limits of agreement (i.e. $\mathrm{P}<0.05$ for two-tailed non-directional test). The limit of agreement is selected to be similar to the Paired Student's t-test for consistency in the statistical validation of mean and median of HR measured.

In order to obtain the value of $\mathrm{W}$, the difference between MMSB and ECG in Table I was calculated. These differences were ranked without regard to the sign of the difference (i.e., rank order the absolute differences). All zero differences were ignored (i.e., pairs with equal members). For differences with equal absolute values (i.e. ties), the same rank was assigned by taking the mean of the rank numbers that would had been assigned if they are different.

Upon completion of the ranking, the original signs on the differences were affix to the rank numbers. All positive ranks $(\mathrm{W}+)$ and all negative ranks (W-) were summed separately. At the same time, the total number of non-zero pairs (n) is recorded.

From the sum of the positive and negative ranks, the variation of the median for the sum of the positive difference was $\mathrm{W}+=273$ and the sum of the negative difference was $\mathrm{W}$ $=393$. The test statistic $\mathrm{W}$ was obtained from the minimum of $\mathrm{W}+$ and $\mathrm{W}$ - (i.e. $\mathrm{W}=273$ ).

The standard deviation (4) for the measured pairs was used together with the mean, $\mu_{w}\left(=0\right.$ for $n>30$ [17]) for the $Z_{w}$ score as shown in (5).

$$
\sigma_{W}=\sqrt{((n *(n+1) *(2 n+1)) / 6)}
$$

where

$\sigma_{W}=$ standard deviation for the sampling distribution of $\mathrm{W}$ (i.e. $\sigma_{W}=127.30$ )

$\mathrm{n}=$ number of pairs of non-zero difference between MMSB and ECG measurements (i.e. $\mathrm{n}=36$ )

$$
Z_{W}=\left(\left(\left(W-\mu_{W}\right) \pm 0.5\right) / \sigma_{W}\right)=\left((W \pm 0.5) / \sigma_{W}\right)(5)
$$
where

$Z_{W}=$ standard deviation for the sampling distribution of $\mathrm{W}$ (i.e. $Z_{\mathrm{w}}=2.148,2.141$ )
To test for the Level of Significance, the table of critical values of $Z_{\mathrm{w}}$ (i.e. Table III [17]) was used and it can be observed that the value of $Z_{\mathrm{w}}=2.148,2.141$ was significant just a shade beyond the 0.025 level for a directional test. For a two-tailed non-directional test, it would be significant just beyond the 0.05 level (i.e. $95 \%$ limits of agreement).

TABLE III: CRITICAL VALUES OF $\pm Z_{\mathrm{w}}[17]$

\begin{tabular}{|c|c|c|c|c|}
\hline \multicolumn{5}{|c|}{ Level of Significance for } \\
\hline \multicolumn{5}{|c|}{ Directional Test } \\
\hline \multicolumn{5}{|c|}{ Non-Directional Test } \\
\hline-- & 0.025 & 0.01 & 0.005 & 0.0005 \\
\hline \multicolumn{5}{|c|}{$\mathrm{Z}_{\mathrm{w}, \text { critical }}$} \\
\hline 1.645 & 1.960 & 2.326 & 2.576 & 3.291 \\
\hline
\end{tabular}

In conclusion, the Wilcoxon Signed-Rank test shows that there was no significant difference at $95 \%$ limits of agreement (i.e. two-tailed non-directional test) between the median of the HR measured by both MMSB and ECG methods. This result suggests that the difference between the two sets of measurement was statistically significant. As such, the Wilcoxon Signed-Rank test supports the use of MMSB as a viable alternative to ECG for HR measurement.

\section{Bland and Altman Test}

The Bland and Altman test (a.k.a. Limits of Agreement) is used in scientific validation on the measurement outputs of an alternative instrument (MMSB) against a "gold standard" (ECG) to determine the limits of agreement for the errors between the two sets of data [18]-[19]. The Bland and Altman method calculates the mean difference between MMSB and ECG methods of measurement (the 'bias'), and 95\% limits of agreement as the mean difference using (6) (or $2 *$ Standard Deviation - SD) [or more precisely $(1.96 * \mathrm{SD})$ ]. It is expected that the $95 \%$ limits include $95 \%$ of differences between the two measurement methods.

$$
S D=\sqrt{\Sigma\left(D_{i}-\bar{D}\right)^{2} /(N-1)}
$$

where

$D=$ difference between instantaneous HR obtained from MMSB and ECG

$\bar{D}=$ mean of the difference between HR obtained from MMSB and ECG

$N=$ total number of HR measurement samples (i.e. $\mathrm{n}=40$ )

The plot is commonly called a Bland-Altman plot and the associated method is usually called the Bland-Altman method [18]. The Bland and Altman method was applied on the measurements from Table I and plotted as shown in Fig. 8. This analysis plots the difference (error) between the two HR measurements with respect to the means between the two scores. In addition, such a plot allows the assessment for systematic bias in the scores between the two systems.

In clinical test, the magnitude of the error at $\pm 2 * \mathrm{SD}$ is used to determine the clinical and practical significance of the measurement errors between the two systems. The magnitude of error should not be clinically or practically significant before the alternative device is accepted as a replacement for the gold standard measurement device.

In the current analysis, the mean and SD of the difference in HR measured between the ECG and the MMSB methods was $-0.05 \pm 0.24 \mathrm{bpm}$ (i.e. $\pm 0.48 \mathrm{bpm}$ based on $\pm 2 *$ SDI. The error data points were found to be evenly distributed across 
the range of heart rate measured, suggesting that there is no systematic error in the measurement. The $\pm 2 * S D$ of $0.48 \mathrm{bpm}$ was found to be practically not significant for measurement of resting HR, suggesting that the MMSB is a suitable alternative for the ECG system for HR measurement. For example, a resting HR of $70 \mathrm{bpm}$ measured with the ECG system, would range between $69 \mathrm{bpm}$ and $71 \mathrm{bpm}$ in the HR measured by the MMSB system. Such a variation ( $\pm 1 \mathrm{bpm})$ is within the normal range of variation in HR measured in healthy individuals.

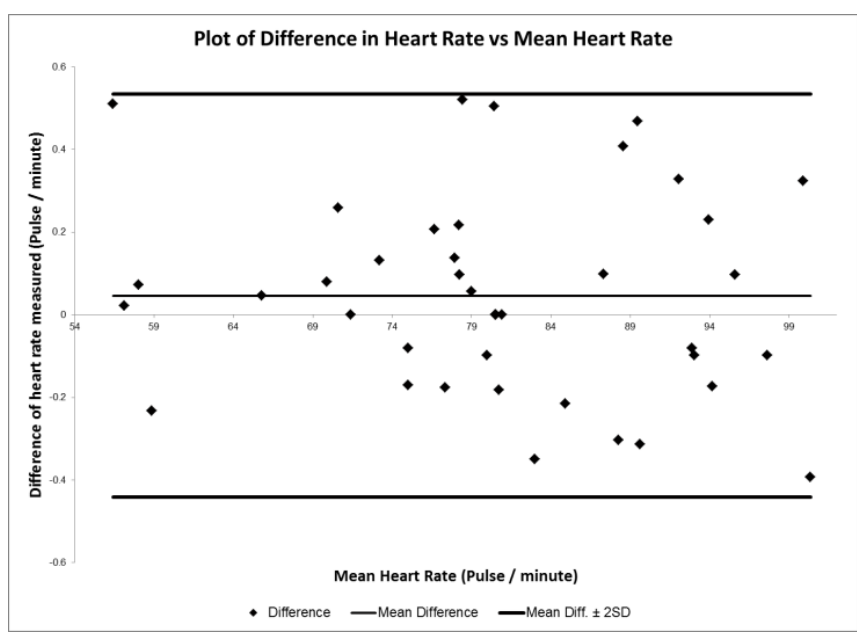

Fig. 8. Plot of Bland and Altman test on HR obtained from MMSB and ECG waveforms

\section{CONCLUSION}

From this study, the concurrent acquisition and measurement of resting HR using a gold standard (ECG) and MMSB on 40 independent measurements were successfully concluded with statistical analyses done on the measured HR. The results of the statistical analyses shows that HR measured by the MMSB was almost perfectly correlated with HR measured with the ECG. This affirms HR measured from both MMSB and ECG methods to be highly interrelated.

The difference between the mean and median of the HR obtained from the two methods (or instrumentations) were also found to be statistically not significant, indicating the use of either methods will deliver HR measurements that are statistically similar.

In addition, the accuracy of HR measured by both methods is found to be in very good agreement for all measurements.

As such, conclusions obtained from each of the 4 statistical tests support the use of the MMSB as a viable alternative to replace ECG in measuring instantaneous HR of healthy individuals under resting condition.

\section{FUTURE WORK}

With the scientific validation of HR completed for MMSB with a gold standard instrumentation, the research work will continue to focus on the use of the accuracy provided by the HR measurement for MMSB to assess the heart rate variability of human subjects.

The objective of such a study is to enable applications to be developed for the monitoring, assessment and management of the health of an individual.

\section{ACKNOWLEDGEMENTS}

The authors would like to thank Nanyang Polytechnic of Singapore and University of Paris Est. for the opportunity to work on this research and development. In addition, the authors would like to thank all volunteers that had supported the scientific validation through their participation in the measurements. In particular, the authors would like to express their gratitude to the School of Engineering (Electronics), Nanyang Polytechnic of Singapore for the usage of facilities and equipment that made this work possible.

\section{REFERENCES}

[1] S. Bowbrick and A. N. Borg, Edinburgh ECG complete, New York, Churchill Livingstone, 2006.

[2] S. M. Burns, AACN protocols for practice: noninvasive monitoring, Jones and Bartlett Publishers, 2006.

[3] S. Yamada, M. Chen, and V. Lubecke, "Sub-uW Signal Power Doppler Radar Heart Rate Detection," in Proceedings of Asia-Pacific Microwave Conference, 2006.

[4] G. Amit et al., "Automatic extraction of physiological features from vibro-acoustic heart signals: correlation with echo-doppler,' Computers in Cardiology, Issue September 25-28, pp. 299-302, 2005.

[5] J. L. Jacobs, P. Embree, M. Glei, S. Christensen, and P. K. Sullivan, "Characterization of a Novel Heart and Respiratory Rate Sensor," in Proceedings of the 26th Annual International Conference of the IEEE EMBS, 2004.

[6] J. Malmivuo and R. Plonsey, "Bioelectromagnetism - Principles and Applications of Bioelectric and Biomagnetic Fields," New-York, Oxford University Press, 1995.

[7] C. T. Phua, G. Lissorgues, and B. Mercier, "Noninvasive acquisition of Blood Pulse using magnetic disturbance technique," International Conference on BioMedical Engineering (ICBME2008)

[8] D. C. McKenzie Md. Research Report - MIO ${ }^{\mathrm{TM}}$ Lifestyle Watch. [Online]. Available http://www.mioglobal.com/files/file/Research\%20Report\%20on\%20 MIO\%20Accuracy.pdf

[9] P. S. Myles, R. McRae, I. Ryder, J. O. Hunt, and M. R. Buckland. "The association between oxygen delivery and consumption in patients undergoing cardiac surgery. Is there supply dependence?" Anaesth Intensive Care 1996; vol. 24, pp. 651-7

[10] P. S. Myles, D. A. Story, and M. A. Higgset al., "Continuous measurement of arterial and end-tidal carbon dioxide during cardiac surgery: Pa-ETCO2 gradient," Anaesth Intensive Care vol. 25, pp. 459-463, 1997.

[11] H. Opdam, L. Wan, and R. Bellomo. "A pilot assessment of the FloTrac(TM) cardiac output monitoring system." Intensive Care Med vol. 33, pp. 344-349, 2007

[12] D. J. Niedhart, H. A. Kaiser, E. Jacobsohn, C. B. Hantler, A. S. Evers, and M. S. Avidan, Intrapatient reproducibility of the BISxp monitor. Anesthesiology vol. 104, pp. 242-248, 2006.

[13] R. E. Anderson, U. Sartipy, and J. G. Jakobsson, "Use of conventional ECG electrodes for depth of anaesthesia monitoring using the cerebral state index: a clinical study in day surgery," Br J Anaesth vol. 98, pp. 645-648, 2007.

[14] D. Button, L. Weibel, O. Reuthebuch, M. Genoni, A. Zollinger, and C. K. Hofer, "Clinical evaluation of the FloTrac/VigileoTM system and two established continuous cardiac output monitoring devices in patients undergoing cardiac surgery," Br J Anaesth vol. 99, pp. 329-336, 2007.

[15] Ed. D. Jim Higgins, The Radical Statistician, 5th Edition

[16] O'Connor, J. John, Robertson, and F. Edmund, "Student's t-test," MacTutor History of Mathematics archive, University of St Andrews, http://www-history.mcs.st-andrews.ac.uk/Biographies/Gosset.html

[17] R. Lowry. Concepts and Applications of Inferential Statistics. Subchapter 12a: The Wilcoxon Signed-Rank Test. [Online]. Available: http://faculty.vassar.edu/lowry/webtext.html

[18] J. Martin Bland and G. Douglas Altman, "Statistical methods for assessing agreement between two methods of clinical measurement," International Journal of Nursing Studies, vol. 47, pp. 931-936, 2010.

[19] D. G. Altman and J. M. Bland, "Measurement in medicine: the analysis of method comparison studies," Statistician 32, pp. 307-317, 1983.

[20] T. C. Pilkington and R. Plonsey, "Engineering Contributions to Biophysical Electrocardiography," 248 pp. IEEE Press, John Wiley, New York, 1982. 
[21] K. H. Zou, K. Tuncali, and S. G. Silverman, "Correlation and Simple Linear Regression," Radiology vol. 227, pp. 617-628, 2003.

[22] E. Whitley and J. Ball, Statistics review 6: Nonparametric methods, Critical Care 2002, vol. 6, pp. 509-513 (DOI 10.1186/cc1820)

[23] E. Zomer, A. Owen, D. J. Magliano, D. Liew, and C. Reid, "Validation of two Framingham cardiovascular risk prediction algorithms in an Australian population: the 'old' versus the 'new' Framingham equation," European Journal of Cardiovascular Prevention and Rehabilitation vol. 18, no. 1, pp. 115-120, The European Society of Cardiology 2011

[24] D. Machin, M. Campbell, P. Fayers, and A. Pinol, "Sample Size Tables for Clinical Studies," Second Ed. Blackwell Science IBSN 0-86542-870-0 pp. 71-72, 2003.

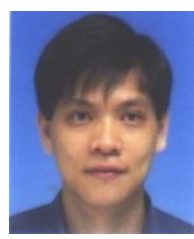

Chee Teck Phua, born in Singapore in March 1971, is a lecturer at the Nanyang Polytechnic of Singapore, School of Engineering (Electronics) since 2004. He has obtained the Bachelor of Electrical and Electronics Engineering $\left(1^{\text {st }}\right.$ Class Honor) in 1996 and Master of Engineering in 2000 from the Nanyang Technological Unversity, Singapore. $\mathrm{He}$ is currently doing his $\mathrm{PhD}$ with the University Paris Est on the topic "Développement d'une nouvelle méthode de mesure du rythme cardiaque et du débit sanguin fondée sur les perturbations localisées d'un champ magnétique". His main fields of research interest include biosensors, smart and assistive solutions and mixed-signal integrated circuit design.

Chee Teck is a member of IEEE and has various publications in international conferences. In addition, he has filed 4 patents in Malaysia, Canada, Australia, India, Korea, Japan, Europe (27 States), Hong Kong, Vietnam, China, USA and Singapore with 3 of these patents granted in Singapore.

$\mathrm{He}$ is currently holding the appointment of Manager in Nanyang Polytechnic, School of Engineering (Electronics) and is actively leading the research and development work on biosensors and product designed to enhance the quality of life for the elderly.

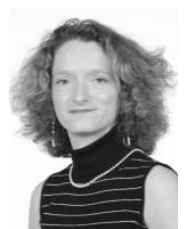

Gaelle Lissorgues, born in France in August 1970, is a Professor at ESIEE in electrical engineering since 1997 obtained the diploma from ENS of Cachan in Applied Physics in 1993 (Agregation), her PhD from University Paris 6 on Laser range finding techniques in 1997 and the "Habilitation à Diriger les Recherches" from University Paris Est on MEMS in 2006. Her main fields of research interest include firstly RF functions integration into micro-systems, applied to wireless sensor networks, and secondly applications of micro-technologies to Biosciences and Medicine i.e. MEMS and micro-sensors for biological, bio-chemical and medical applications.

She has written 6 patents and published more than 25 papers in international journals and more than 85 conference papers in international conferences.
She is member of the research laboratory ESYCOM (EA 2552), and participates to several committees like the AVIESAN on medical technologies. She is currently supporting $4 \mathrm{phD}$ students, and previously followed $11 \mathrm{phD}$ students. She is also involved in several national projects (from the French Research Agency) and European projects with industrial partnerships (THALES, COVENTOR, NXP).

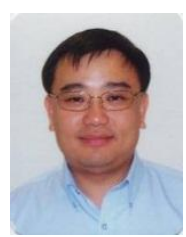

Boon Chong Gooi, graduated from the National University of Singapore in 1989 with a Bachelor of Engineering (First Class Honours) degree in Mechanical Engineering. Boon Chong subsequently obtained his Master of Engineering degree from the Nanyang Technological University in 1992 with specialization in Computational Fluids Dynamics. In 1992, Boon Chong joined Nanyang Polytechnic (NYP) after having worked in the electronics industry for three years. Presently, he is the manage responsible for the Applied Research Group in the School of Engineering (Electronics). His key functions include overseeing the strategies and areas of applied research that have technological, economic and social impact to the local and international industries and the society.

With a wealth of academic and research experience that span twenty years, Boon Chong is currently spearheading NYP effort in the Research and Development (RandD) of innovative technologies and solutions that address the issues of the aging population in Singapore. This area of research inspired Boon Chong to pursue studies in psychological sciences and he obtained his Specialist Diploma in Applied Psychology in 2009 and his Graduate Diploma in Psychology and Counselling in 2010 from The Adventis School of Management in collaboration with the City University of New York (CUNY). Since then, Boon Chong area of interest is in clinical psychology research and statistical analysis. Boon Chong is the inventor/co-inventor of six patents to date. In addition, Boon Chong is a senior member of the Institution of Engineer, Singapore.

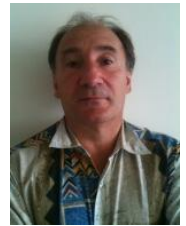

Bruno Mercier was born in 1960 in Le Mans, France. He graduated from CNAM (Conservatoire National des Arts et Metiers) in Paris where he obtained the Diplome d'Etudes Supérieurs Techniques (equivalent of BS) in 1986, and the Diplôme d'Ingénieur (equivalent of Msc degree) in 1988, both from the Department of Physics, microelectronics. semiconductors and vacuum systems for

He is currently the Director of the cleanroom facilities of ESIEE Paris, a high school of Electrical Engineering, where he leads a group of 10 people. Bruno Mercier developed an expertise in power integrated circuits and in micro technologies for MEMS and biosensors manufacturing since 1997 . He established numerous industrial partnerships for RandD. His research interests are based on optical tools, MEMS and microfluidics. Bruno Mercier is co-author of more than 20 publications in scientific journals. He is also involved in cleanroom training and consultancy for establishing cleanroom facilities. 